\title{
DDR2 receptor promotes MMP-2-mediated proliferation and invasion by hepatic stellate cells
}

\author{
Elvira Olaso, ${ }^{1}$ Kazuo Ikeda, ${ }^{1}$ Francis J. Eng, ${ }^{1}$ Lieming Xu, ${ }^{1}$ Li-Hsien Wang, ${ }^{2}$ \\ Hsin Chieh Lin, ${ }^{2}$ and Scott L. Friedman ${ }^{1}$ \\ ${ }^{1}$ Division of Liver Diseases and Department of Medicine, Mount Sinai School of Medicine, New York, New York, USA \\ ${ }^{2}$ Regeneron Pharmaceuticals, Tarrytown, New York, USA \\ Address correspondence to: Scott L. Friedman, Box 1123, Mount Sinai School of Medicine, 1425 Madison Ave, Room 1170F, \\ New York, New York 10029, USA. Phone: (212) 659-9501; Fax: (212) 849-2574; E-mail: frieds02@doc.mssm.edu. \\ Elvira Olaso's present address is: Biomedical Research and Technological Development Institute, INBIOMED Foundation, \\ San Sebastian Technology Park, Gipuzkoa, Spain. \\ Lieming Xu's present address is: Shanghai University of TCM, Shanghai, China. \\ Received for publication January 30, 2001, and accepted in revised form September 14, 2001.
}

Type I collagen provokes activation of hepatic stellate cells during liver injury through mechanisms that have been unclear. Here, we tested the role of the discoidin domain tyrosine kinase receptor 2 (DDR2), which signals in response to type I collagen, in this pathway. DDR2 mRNA and protein are induced in stellate cells activated by primary culture or in vivo during liver injury. The receptor becomes tyrosine phosphorylated in response to either endogenous or exogenous type I collagen, whereas its expression is downregulated during cellular quiescence induced by growth on Matrigel. We developed stellate cell lines stably overexpressing either wild-type DDR2, a constitutively active chimeric DDR2 receptor (Fc-DDR2), a truncated receptor expressing the extracellular domain, or a kinase-dead DDR2 Cells overexpressing DDR2 showed enhanced proliferation and invasion through Matrigel, activities that were directly related to increased expression of active matrix metalloproteinase 2 (MMP-2). These data show that DDR2 is induced during stellate cell activation and implicate the phosphorylated receptor as a mediator of MMP-2 release and growth stimulation in response to type I collagen. Moreover, type I collagen-dependent upregulation of DDR2 expression establishes a positive feedback loop in activated stellate cells, leading to further proliferation and enhanced invasive activity.

J. Clin. Invest. 108:1369-1378 (2001). DOI:10.1172/JCI200112373.

\section{Introduction}

Hepatic fibrosis, or scar deposition in response to chronic injury, is similar in all forms of liver disease (1). Accumulation of fibrillar, or type I, collagen occurs in the subendothelial space between hepatocytes and endothelial cells, where it replaces a low-density basement membrane-like matrix containing type IV collagen. This conversion of the subendothelial matrix to one rich in fibrillar collagen is a pivotal event mediating the loss of differentiated function characteristic of progressive liver disease.

Hepatic stellate cells are the major source of fibrosis following their two-stage activation from quiescent vitamin A-rich cells to proliferative and fibrogenic myofibroblasts (1). Initiation of this cascade is ascribed to paracrine stimulation by Kupffer and endothelial cells. In contrast, perpetuation comprises a broad and largely autocrine conversion that includes enhanced proliferation, fibrogenesis, migration, contractility, cytokine release, and production of matrix proteases (2).

The accumulation of type I collagen has a direct activating effect on stellate cells through an unknown mechanism (3). Stellate cell activation is further accelerated by upregulation of matrix metalloproteinase 2 (MMP-2) activity, because this enzyme degrades the normal subendothelial matrix, hastening its replace- ment by fibrillar collagen (4). The activity of MMP-2 is tightly regulated by specific inhibitors, including TIMP-2 (tissue inhibitor of metalloproteinase-2) and by activators including membrane-type matrix metallproteinase-1 (MT1-MMP) $(5,6)$.

Major features of stellate cell activation are mediated by the induction of transmembrane growth factor receptors with kinase activity or receptor tyrosine kinases (RTKs). For example, stellate cell proliferation and migration is preceded by a rapid induction of the $\beta$-PDGF receptor $(7,8)$, as well as by mitogenic responses to epidermal growth factor (9) and fibroblast growth factor (10). To identify additional RTKs induced during stellate cell activation, previously we performed homology PCR using primers derived from the conserved kinase domain of RTKs and a template consisting of activation-specific cDNAs from rat stellate cells (11). This approach yielded several RTKs, including a partial cDNA encoding the discoidin domain receptor 2 (DDR2), also known as Tyro10 or TKT (12-14).

The DDR subfamily (which includes DDR1 and DDR2) has several features that distinguish it from other RTKs. DDRs signal in response to collagens rather than soluble peptide growth factors, and they display a relatively slow onset of phosphorylation, occurring in hours rather than minutes $(15,16)$. The 
triple helical structure of collagen is required for activation of both DDR1 and DDR2 (16). However, DDR1 expression is confined to epithelial tissues where it is activated equally by collagen types I, IV, and V. In contrast, DDR2 is found in mesenchymal cells and is activated primarily by collagen type I, and to a lesser extent, by collagen types II, III, and V $(15,16)$. DDR 2 is also present at high levels in stromal cells surrounding DDR1-expressing epithelial human tumor cells (14). DDR1 mRNA is overexpressed up to threefold in human primary mammary carcinomas compared with adjacent epithelial cells (17).

Stellate cell interactions with collagen have, until now, been ascribed to integrins, a large family of heterodimeric receptors (see refs. 19-21 for review). Activated stellate cells express the collagen-binding integrin receptors $\alpha_{1} \beta_{1}, \alpha_{2} \beta_{1}$, and $\alpha_{6} \beta_{1}(20,21)$. Interestingly, no studies have demonstrated that integrins mediate features of stellate cell activation. In particular, inhibition of $\alpha_{1} \beta_{1}$ and $\alpha_{2} \beta_{1}$ integrins using blocking $A b$ 's does not affect MMP-2 synthesis in stellate cells (22). Thus, some key signal(s) to stellate cell activation in response to type I collagen remains unknown.

Based on the identification of DDR2 mRNA in stellate cells, we have explored the possibility that DDR2 could account for the observed effects of fibrillar collagen on stellate activation both in vivo and in vitro. Our results demonstrate that DDR2 expression and signaling are induced in stellate cells during liver injury and mediate key features of stellate cell activation.

\section{Methods}

Stellate cell isolation and culture. Hepatic stellate cells (HSCs) and Kupffer cells were isolated from retired breeder Sprague-Dawley rats as described previously (23). In brief, the liver was perfused in situ with pronase and collagenase, and the nonparenchymal cells were separated by density gradient centrifugation with Accudenz A.G. (Accurate Chemical and Scientific Corp., Westbury, New York, USA). Stellate cells were recovered from the $8 \%$ Accudenz A.G. layer and cultured on plastic dishes in DMEM supplemented with 10\% FCS (DMEM-FCS). Kupffer cells were purified from the $17 \%$ layer by centrifugal elutriation and cultured for 48 hours in DMEM-FCS. Cells were then washed and maintained in serum-free media for the next 24 hours. The resulting conditioned media was centrifuged briefly and frozen at $-80^{\circ} \mathrm{C}$. Some studies used an immortalized rat stellate cell line, HSC-T6, as described previously (24). HSC-T6 cells were routinely maintained in DMEM-FCS. For some studies, cells were plated at $30 \%$ confluence onto a $0.5-\mathrm{mm}$ layer of Matrigel (Becton Dickinson, Bedford, Massachusetts, USA) or type I collagen (Becton Dickinson).

Models of liver injury. To induce liver injury, animals were administered $0.5 \mu \mathrm{l}$ of $\mathrm{CCl}_{4}$ per gram of body weight by gavage (25) or underwent ligation of the common bile duct (26). Control animals received corn oil by gavage or underwent sham laparotomy, respectively.
Northern blot analysis. Total RNA was extracted with RNeasy (QIAGEN Inc., Valencia, California, USA) from cells cultured on plastic or from stellate cells immediately following isolation. HSC-T6 cells cultured in Matrigel were released using Matri Sperse (Becton Dickinson), were pelleted by centrifugation, and resuspended in the RNeasy lysis solution. Total RNA was also isolated from frozen liver tissue. Ten micrograms of total RNA was subjected to electrophoresis on a $1 \%$ agarose/formaldehyde gel, transferred to nitrocellulose membrane (Schleicher \& Schuell Inc., Keene, New Hampshire, USA), and hybridized overnight at $42^{\circ} \mathrm{C}$ with ${ }^{32} \mathrm{P}-$-labeled cDNA probes for either rat collagen $\alpha 1$ (I) (27) or rat DDR2 (nucleotides 2512-3255) (11). Blots were washed three times at $65^{\circ} \mathrm{C}$ in $2 \times \mathrm{SSC} / 0.1 \%$ SDS, exposed to a phosphor storage screen, and scanned in a Molecular Dynamics (Sunnyvale, California, USA) Phosphorimager. Results were normalized to GAPDH mRNA using the ImageQuant software (Molecular Dynamics.

DDR2 immunodetection. Immunoprecipitation/Western blot analysis for DDR2 was performed using a rabbit polyclonal Ab, R2-JM, raised against the juxtacrine domain of mouse DDR2 (amino acid [aa] 429-557). Primary rat stellate cell cultures maintained in DMEM-FCS for up to 8 days were lysed in radio immunoprecipitation assay (RIPA) buffer containing protease inhibitors (Roche Molecular Biochemicals, Indianapolis, Indiana, USA), $2 \mathrm{mM}$ sodium orthovanadate, and $10 \mathrm{mM}$ sodium fluoride. In some experiments primary stellate cells activated by culture on plastic for 10 days were cultured in Matrigel for up to 96 hours, released using matrisperse, and pelleted by centrifugation before cell lysis. Lysate $(250 \mu \mathrm{g})$ was incubated with R2-JM polyclonal anti-DDR2 Ab for 1 hour at $4^{\circ} \mathrm{C}$. Protein A-Sepharose beads (Sigma Chemical Co., St. Louis, Missouri, USA) were added for 1 hour at $4^{\circ} \mathrm{C}$, then precipitated by centrifugation, and washed three times with RIPA buffer. The protein ASepharose-bound proteins were extracted by 10 -minute incubation in Laemmli buffer at $80^{\circ} \mathrm{C}$. COS-1 African green monkey kidney cells (COS) cells were transfected with the DDR2 constructs (see below) and lysed in $2 \times$ Laemmli buffer. Protein aliquots $(80-300 \mu \mathrm{g})$ were separated through $8 \%$ SDS-PAGE electrophoresis and blotted onto a nitrocellulose membrane (Bio-Rad Laboratories, Hercules, California, USA). DDR2 was detected using the R2-JM anti-DDR2 Ab (1:1,000) and horseradish peroxidase-conjugated protein A $(1: 20,000)$. Phosphorylated DDR2 was detected using the monoclonal anti-phosphotyrosine Ab 4G10 (1:20,000; Upstate Biotechnology Inc., Lake Placid, New York, USA) and horseradish peroxidase-conjugated goat anti-mouse IgG $(1: 20,000)$. The bands were visualized using the Super Signal Femto Substrate kit (Pierce Chemical Co., Rockford, Illinois, USA).

Western blot. Stellate cells were cultured for up to 48 hours in the presence or absence of $0.5 \mathrm{mM}$ cis-4hydroxy-L-proline (cis-OH-Pro; Sigma Chemical Co.) in DMEM without FCS. Cell viability was checked by trypan blue exclusion. Cells were lysed in RIPA buffer as 
described above. Media was harvested and briefly centrifuged. Equal amounts of protein were resolved on $6 \%$ SDS-PAGE electrophoresis, blotted onto a nitrocellulose membrane (Bio-Rad), and incubated with a polyclonal $\mathrm{Ab}$ against the $\alpha 1(\mathrm{I})$ chain of human type I collagen (kindly provided by D. Schuppan, Universitatsklinikum Benjamin Franklin, Berlin, Germany), followed by antirabbit IgG $(1: 5,000)$. To detect $\alpha$-smooth muscle actin (ASMA) and tubulin expression, $25 \mu \mathrm{g}$ of cell lysate were separated through $10 \%$ SDS-PAGE electrophoresis, blotted onto a nitrocellulose membrane (Bio-Rad), and incubated with mAb's against ASMA or tubulin (1:1,000; Sigma Chemical Co.) and anti-mouse IgG $(1: 5,000)$. In some experiments, membranes were also incubated in 1:500 polyclonal anti-MT1-MMP Ab (Chemicon International, Temecula, California, USA) that recognized its latent and active forms, followed by anti-rabbit IgG $(1: 5,000)$. The bands were visualized using the enhanced chemiluminescence (ECL) system (Amersham Pharmacia Biotech, Uppsala, Sweden).

Generation of stable stellate cell lines expressing DDR2 or DDR2 mutants. To stably overexpress DDR2 and its mutants, subconfluent HSC-T6 cultures were infected with a retrovirus containing a bi-cistronic construct encoding either the wild-type (14) or mutant mouse DDR2 cDNAs (described below) under control of the viral long terminal repeat (LTR). The retrovirus plasmid was derived from the plasmid LZRSPBMN-LacZ in which the LacZ coding sequences were replaced with a multiple cloning site immediately downstream of the packing signal. Following these sequences was a cassette encoding enhanced green fluorescence protein (GFP) under the control of an internal ribosomal entry site (IRES).

To generate cell lines expressing mutant DDR2 constructs, the following cDNAs were subcloned downstream of the viral LTR: wild-type DDR2 (wt-DDR2), the full-length mouse cDNA sequence with a Myc tag at the carboxyl terminus; constitutively active DDR2 (FcDDR2), a constitutively dimerized DDR kinase resulting from the replacement of the DDR2 extracellular domain (aa 1-99) with the $\mathrm{Fc}$ region of human IgG1; kinase-dead DDR2 (kd-DDR2), a threonine $\rightarrow$ alanine mutation at aa 664 generated by site-directed mutagenesis; and ec-DDR, the mouse cDNA expressing aa $1-443$, lacking the kinase domain but retaining the extracellular and transmembrane domains. Retroviral vector plasmids were transfected into the Phoenix-A line by the calcium phosphate method (Invitrogen Life Sciences, Carlsbad, California, USA). Puromycin-containing medium $(1 \mu \mathrm{g} / \mathrm{ml})$ was used to select for cells containing the retroviral vector plasmid. Virus was harvested by filtering medium through a low proteinbinding $0.45-\mu \mathrm{m}$ filter, then aliquoted. For infections, $2 \times 10^{6}$ cells per 10 -cm dish were plated 24 hours before infection. Each plate was infected with $10 \mathrm{ml}$ of 1:10 dilution of the virus stock. Cells with high GFP expression were recovered by FACS sorting to GFP (MoFlo; Cytomation, Fort Collins, Colorado, USA). Then cells were grown in monolayer culture overnight and frozen.
After cell sorting, stellate cells were grown in monolayer culture overnight, then frozen and used within 48 to 72 hours after thawing. Only cells in the first passage after thawing were used for all experiments, during which time DDR2 expression remained stable, as assessed by flow cytometry for GFP expression and Western blot for DDR2. Control cells were infected with a retroviral vector expressing only the internal ribosomal entry site driving the GFP cDNA.

Cell growth assays. Stellate cells were maintained 42 hours in DMEM plus 10\% FCS and labeled for 6 hours with $\left[{ }^{3} \mathrm{H}\right]$-thymidine $(10 \mu \mathrm{Ci} / \mathrm{mL}$ in DMEM). Parallel cultures of cells were trypsinized and counted directly using a hemacytometer. In some experiments, stellate cells were cultured for up to 42 hours in DMEM supplemented with $1 \%$ FCS in the presence of increasing concentrations of recombinant TIMP-2 (Biogenesis Inc., Kingston, New Hampshire, USA and R\&D Systems, Inc., Minneapolis, Minnesota, USA) or the synthetic MMP inhibitor GM6001 (Chemicon International). At the concentrations used $(0-25 \mu \mathrm{g} / \mathrm{ml}$ recTIMP- 2 and $0-10$ $\mu \mathrm{M}$ GM6001 for a maximal incubation time of 24 hours), none of them affected cell viability as assessed by trypan blue exclusion. Media was then removed from the culture wells and fresh DMEM containing $\left[{ }^{3} \mathrm{H}\right]$-thymidine was added for another 6 hours. Cells were washed three times in PBS to remove nonincorporated isotope and fixed in $100 \%$ methanol. The $\left[{ }^{3} \mathrm{H}\right]$-thymidine incorporated into acid-insoluble material was extracted with $\mathrm{NaOH}$ and measured in a liquid scintillation beta-counter. In some experiments, cell proliferation was measured using the nontoxic alamarBlue assay (BioSource International, Camarillo, California, USA), according to the manufacturer's instructions. In brief, $10 \mu \mathrm{l}$ of alamarBlue was added to 96 -well plates containing $5 \times 10^{3}$ cells $/ 100 \mu$ l of DMEM plus 10\% FCS. Absorbance at 595 $\mathrm{nm}$ and $575 \mathrm{~nm}$ was measured every 4 hours using a Perkin Elmer HTS700-Plus plate reader (Applied Biosystems, Foster City, California, USA). Results were expressed as percentage of reduced alamarBlue, according to manufacturer's specifications.

Analysis of MMP-2. Stellate cells (50-70\% confluent) were washed four times with PBS and maintained for 12 or 24 hours in serum-free medium. The media was then harvested, briefly centrifuged, and immediately frozen at $-20^{\circ} \mathrm{C}$ to prevent autoactivation of MMP-2. Aliquots from identical numbers of HSC-T6 cells were later analyzed by gelatin zymography on $8 \%$ polyacrylamide gels, as described previously (28). Results were quantified by scanning densitometry analysis using the ImageQuant software. Gelatinase activity was also analyzed using a biotinylated gelatinase substrate, which is cleaved by active gelatinase enzymes. Remaining biotinylated fragments were added to a biotin-binding plate and detected as streptavidin-binding complex detected at OD $450 \mathrm{~nm}$ $\left(1 / \mathrm{OD}_{450}\right.$; Chemicon International). To activate MMP-2, conditioned media were incubated 2 hours at $37^{\circ} \mathrm{C}$ with 2.5 mM APMA ( $p$-aminophenylmercuric acetate; Sigma Chemical Co.). For Western blot analysis of MMP-2, the 


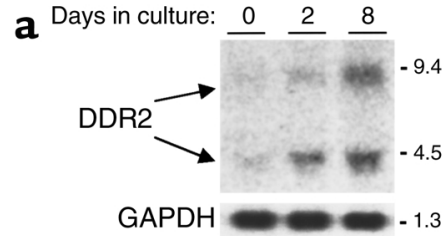

C Days in culture:
DDR2 $\underset{\text { IP: } \alpha \text { DDR2 }}{\rightarrow} \frac{0}{-116} \quad$ IB: $\alpha$ DDR2

d

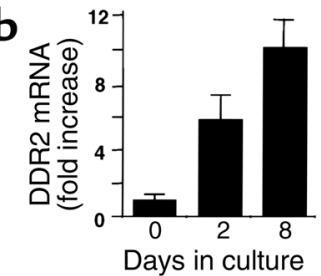

IB: $\alpha$-tubulin

\section{Figure 1}

DDR2 is induced during stellate cell activation in vitro. Stellate cells isolated from normal rat livers were maintained in monolayer culture for up to 8 days, and DDR2 mRNA and protein were analyzed. (a) Northern blot analysis performed with $10 \mu \mathrm{g}$ mRNA from primary cultures of stellate cells hybridized to DDR2 and GAPDH cDNAs. (b) Summary histogram showing the induction of DDR2 during stellate cell activation by in vitro culture based on data from $\mathbf{a}$. Results are expressed as a ratio of DDR2 mRNA versus GAPDH mRNA. (c) Stellate cells were cultured for 0,2 , and 8 days, lysed in RIPA buffer, immunoprecipitated, immunoblotted, and probed with the polyclonal anti-DDR2 Ab R2-JM. COS-1 cells were transfected with fulllength DDR2 $(+)$ or empty vector $(-)$. (d) Lysates were also analyzed by Western blot for tubulin as a control for protein loading.

serum-free conditioned medium harvested from the stellate cell cultures was concentrated 20-fold (Centriplus concentrators, $10 \mathrm{kDa}$ MW cutoff; [Millipore, Bedford, Massachusetts, USA]), electrophoresed through $12 \%$ SDS-PAGE, and transferred to nitrocellulose membranes. Blots were blocked with $5 \%$ nonfat milk and incubated overnight at $4^{\circ} \mathrm{C}$ in 1:500 diluted $\mathrm{mAb}$ against latent MMP-2 (Neomarkers, Lab Vision Corp., Fremont, California, USA) and 1 hour with 1:5,000 dilution of rabbit anti-mouse $\mathrm{Ab}$ (Amersham). In some experiments, membranes were also incubated in 1:500 diluted polyclonal anti-TIMP-2 Ab (Chemicon International), followed by 1:5,000 dilution of goat anti-rabbit $\mathrm{Ab}$ (Amersham). The bands were visualized using the Super Signal Femto Substrate kit (Pierce Chemical Co.).

Cell migration through Matrigel. Cell migration assays were performed as described previously (29) with minor modifications. Culture inserts with $8-\mu \mathrm{m}$ pores coated with $100 \mu \mathrm{g} / \mathrm{cm}^{2}$ Matrigel were placed on top of 24-well plates (Becton Dickinson). Cells $\left(2 \times 10^{4}\right)$ suspended in DMEM supplemented with $1 \%$ FCS were placed on the upper insert. Conditioned media from Kupffer cell cultures supplemented with $1 \%$ FCS were used as chemoattractant in the lower compartment. In some experiments cells were pretreated with $20 \mu \mathrm{M}$ GM6001 for 1 hour, then placed in the invasion chamber in the presence of $10 \mu \mathrm{M}$ GM6001. Cells were allowed to migrate for up to 26 hours. Nonmigrated cells were removed with a cotton swab. Cells that migrated and adhered to the underside of the membrane were fixed in $100 \%$ methanol, stained with
$0.2 \%$ crystal violet, and counted in ten random, highpower fields per membrane. Data were expressed as migration relative to values obtained in control cells at each time point.

Statistics. Results refer to mean plus or minus SEM. Comparisons among groups were done with the Student $t$ test, and $P$ values less than 0.5 were considered significant. Each experiment was performed in duplicate or triplicate and repeated at least two times with independent samples derived from at least two rat livers per time point, two independent primary cultures, or two independent retroviral infections.

\section{Results}

$D D R 2$ is induced during stellate cell activation in culture and in vivo. Our previous studies identified DDR2 mRNA among several RTK transcripts expressed by activated rat stellate cells (11). To document induction of DDR2 mRNA and protein during stellate cell activation, cells were isolated from normal rat liver and cultured on uncoated plastic for up to 8 days; this condition mimics key features of stellate cell activation observed in vivo (3). Transcripts of 4.5 and $9.4 \mathrm{~kb}$ increased after 2 days in culture, with maximal expression after 8 days (Figures 1, a and b). Expression of DDR2 mRNA was not detected in cultured hepatocytes or Kupffer cells. Additionally, no DDR1 mRNA was detected in stellate cells (results not shown).
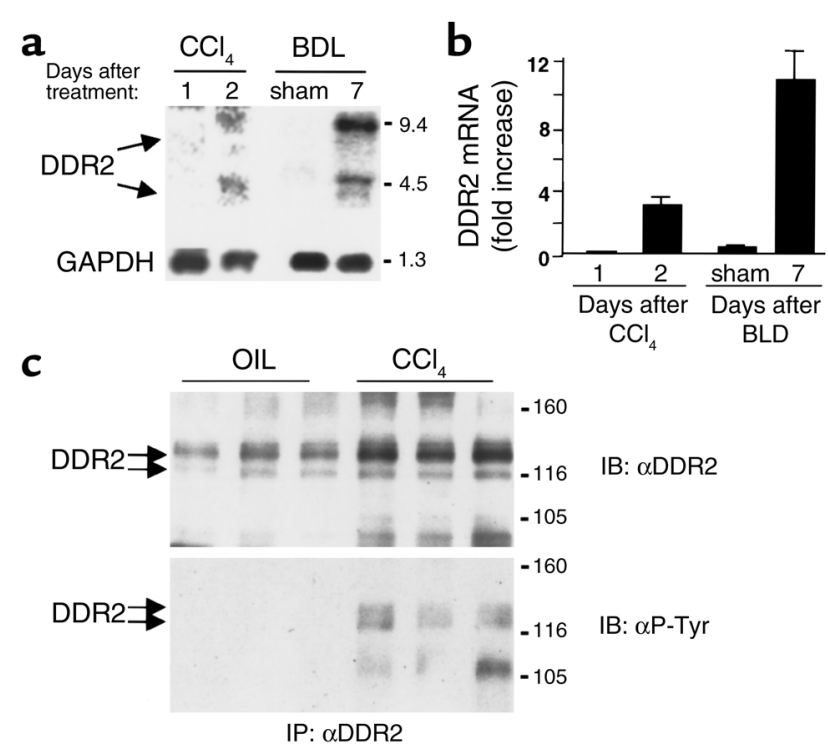

\section{Figure 2}

DDR2 is induced in stellate cells during liver injury. (a) Northern blot analysis of DDR2 mRNA expression in rat stellate cells isolated after administration of a single dose of $\mathrm{CCl}_{4}$ or after bile duct ligation. (b) Summary histogram demonstrating the induction of DDR2 during liver injury. Results are expressed in the histograms as the ratio of DDR2 mRNA versus GAPDH mRNA. (c) Total liver extracts from rats treated 4 weeks with $\mathrm{CCl}_{4}$ or corn oil vehicle alone were lysed in RIPA buffer. DDR2 was immunoprecipitated with the R2-JM Ab, followed by Western blot analysis with an anti-phosphotyrosine $A b$ (4G10) or the anti-DDR2 Ab R2-JM. 


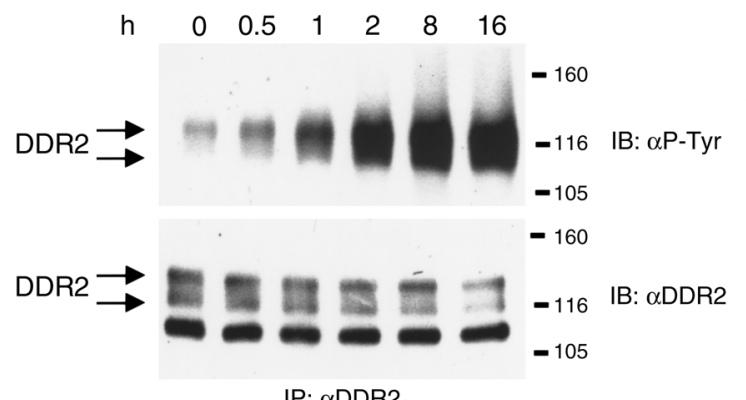

IP: $\alpha$ DDR2

\section{Figure 3}

Type I collagen stimulates DDR2 phosphorylation in stellate cells. HSC-T6 stellate cells were stimulated with $10 \mu \mathrm{g} / \mathrm{ml}$ type I collagen for increasing intervals and lysed in RIPA buffer. DDR2 was immunoprecipitated with the anti-DDR2 Ab R2-JM, followed by Western blot analysis with an antiphosphotyrosine $A b$ (4G10) or anti-DDR2 (R2-JM).

DDR2 protein was induced in a pattern similar to DDR2 mRNA (Figure 1c). Western blot detection using an $\mathrm{Ab}$ raised against the DDR2's juxtamembrane domain (R2JM) revealed two specific bands of approximately 116 and $120 \mathrm{kDa}$, barely apparent after 2 days in culture and readily visible after 8 days. COS cells transfected with fulllength DDR2 cDNA were used as positive controls.

To determine whether DDR2 was induced in stellate cells during acute liver injury in vivo, we analyzed mRNA in cells isolated from rat liver after a single dose of carbon tetrachloride $\left(\mathrm{CCl}_{4}\right)$ or after bile duct ligation (Figure 2). These two mechanistically distinct models of liver injury have been used extensively to characterize stellate cell activation in vivo (30). Maximal DDR2 mRNA expression was observed in stellate cells isolated 48 hours after the dose of $\mathrm{CCl}_{4}$ was administered and persisted after 96 hours. Interestingly, this time point is considerably later than the peak of mRNA for $\beta$-PDGF receptor (8). In animals with ligation of the common bile duct, marked upregulation of DDR2 mRNA was observed after 7 days versus sham-operated control animals.

DDR2 protein expression was analyzed in total liver after 4 weeks of $\mathrm{CCl}_{4}$ administration, a time point at which there is a two- to threefold increase in total liver collagen as assessed by Sirius red staining (result not shown). In these chronically injured animals, a twofold increase in DDR2 expression was detected versus control animals. Furthermore, phosphorylated DDR2 was detected only in injured livers (Figure 2c).

Collagen type I stimulates DDR2 phosphorylation in activated stellate cells. To determine whether collagen I induces DDR2 phosphorylation in stellate cells, soluble type I collagen $(10 \mu \mathrm{g} / \mathrm{ml})$ was added to HSC-T6 cells for up to 16 hours. HSC-T6 cells are an activated rat stellate cell line (24). DDR2 was immunoprecipitated from cell lysates using the anti-DDR2 polyclonal Ab, R2-JM, and phosphorylated DDR2 was detected by Western blot analysis with an antiphosphotyrosine $\mathrm{Ab}$ (4G10). Maximal DDR2 phosphorylation was reached after 2 hours and remained maximal for up to 16 hours (Figure 3 ).
Because activated stellate cells produce abundant type I collagen (31), we examined whether endogenous collagen was sufficient for DDR2 autophosphorylation. As predicted, DDR2 phosphorylation was observed in HSC-T6 cells without adding exogenous type I collagen (Figure 3, lane 1). To confirm a direct requirement for endogenous collagen and not another matrix component, HSC-T6 cells were cultured for 24 and 48 hours in the presence of the proline analogue cis-OH-Pro, which inhibits synthesis of collagen without affecting cellular viability (32). Inhibition of collagen synthesis was confirmed by Western blot analysis of cell lysates using an anticollagen Ab (Figure 4a). Immunoprecipitation of cell lysates with the antiDDR2 Ab R2-JM was followed by Western blot analysis with either R2-JM or an antiphosphotyrosine Ab (4G10). Phosphorylated DDR2 was diminished in cells cultured in the presence of cis-OH-Pro, even though the total levels of DDR2 remained constant (Figure 4b).

The observation that DDR2 was phosphorylated in vivo during liver injury, but not in normal livers (Figure $2 \mathrm{c}$ ), suggests that the increased collagen production during liver injury results in DDR2 phosphorylation.

Extracellular matrix composition regulates DDR2 expression in stellate cells. We demonstrated previously that a basement membrane-like matrix (Matrigel) resembling that found in the normal hepatic subendothelial space preserves a quiescent phenotype of stellate cells, characterized by reduced cell proliferation, spherical morphology, and diminished ASMA expression (3). Therefore, we examined whether this matrix-induced quiescence correlated with levels of endogenous DDR2. Activated, immortalized stellate cells (HSC-T6) were cultured for 3 days on either Matrigel or type I collagen. Compared with cells grown on type I collagen, cells on Matrigel had significantly reduced DDR2 mRNA and protein expression (Figure 5, a and b), which correlat-

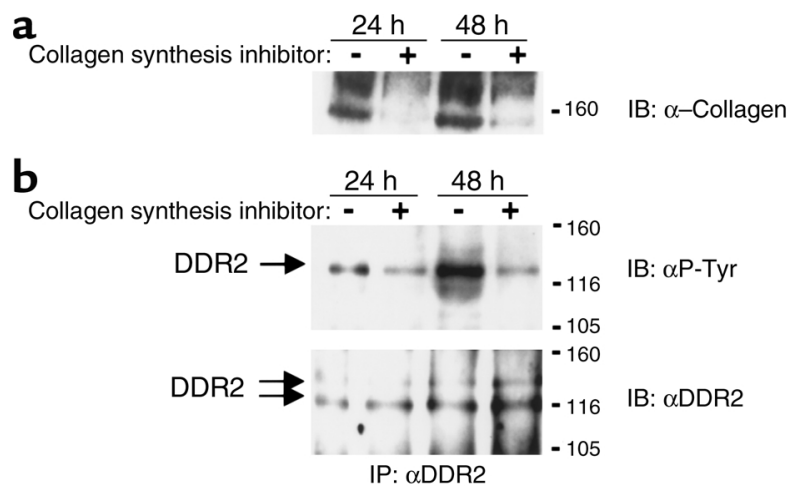

Figure 4

Endogenous collagen synthesis is sufficient for DDR2 phosphorylation. HSC-T6 stellate cells were cultured for 48 hours in the presence of the proline analogue cis-OH-Pro to reduce collagen synthesis. (a) Levels of type I collagen were analyzed with a Western blot of cell lysates using a polyclonal $\mathrm{Ab}$. (b) Cell lysates were immunoprecipitated with the polyclonal anti-DDR2 Ab R2-JM, followed by analysis of Western blotting with an antiphosphotyrosine Ab (4G10) or anti-DDR2 (R2-JM). 

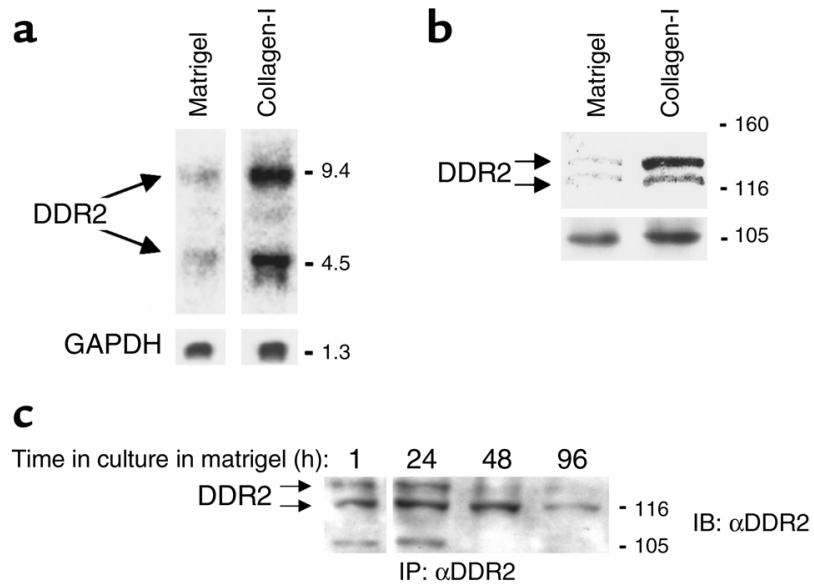

Figure 5

Extracellular matrix regulates DDR2 expression. Levels of DDR2 mRNA (a) and protein (b) expressed by immortalized HSCs (HSC-T6) were analyzed after 3 days of growth on either Matrigel or type I collagen gels. Tubulin or GAPDH mRNA expression levels were assessed, respectively, as loading controls. (c) In primary stellate cells activated by growth in primary culture on plastic, transfer to Matrigel led to diminished DDR2 expression after 48 hours in culture, as assessed by Western blot analysis.

ed with a $70 \%$ decrease in cell proliferation and a $75 \%$ reduction in ASMA, two key functional attributes of activated stellate cells (data not shown). DDR2 downregulation was observed 48 hours after plating and was maximal after 3-5 days in culture. Similar results were observed in activated primary stellate cells transferred to a Matrigel (Figure 5c). To exclude a toxic effect of Matrigel, cells were replated on plastic; after 48 hours, the levels of DDR2 were similar to those observed in cells maintained solely on plastic (not shown).

DDR2 promotes stellate cell proliferation. To explore the functional activity of DDR2, immortalized rat stellate cells (HSC-T6 cells) were infected by a retrovirus encoding either a kinase-dead DDR2 mutant (kd-DDR2), a truncated form lacking the kinase domain but retaining the extracellular and transmembrane domains
(ec-DDR2), or a chimeric molecule in which the native DDR2 extracellular domain was replaced by the Fc portion of immunoglobulin (Fc-DDR2). This chimeric receptor preserved the intracellular domain while provoking spontaneous receptor clustering, thereby enhancing receptor phosphorylation and downstream signaling (see Methods). Each of these retroviral constructs also expressed enhanced GFP under the control of an IRES, which enabled the isolation of infected cells by cell sorting based on GFP fluorescence. As expected, expression of kd-DDR2 inhibited the phosphorylation of the endogenous DDR2 in response to collagen type I by $80 \%$ (Figure 6a). Cellular proliferation was compared between these engineered cell lines and control cells infected with a retrovirus expressing only the IRES and GFP. Two independent measures of cell growth, $\left[{ }^{3} \mathrm{H}\right]$-thymidine uptake and cell number, were used. By either measure, DDR2 activity correlated with cell proliferation, with lowest levels in cells expressing the kdDDR2 and the truncated DDR2 receptor (ec-DDR2), intermediate levels for control cells, and increased growth in stellate cells expressing the constitutively active receptor (Fc-DDR2) (Figure 6, b and c).

DDR2 promotes MMP-2 expression and activity in stellate cells, which stimulates cellular proliferation. In a human fibrosarcoma cell line HT-1080, activation of DDR2 by type I collagen leads to upregulation of MMP-1, which can degrade fibrillar collagens (15). However, stellate cells do not express the interstitial collagenases MMP-1 or MMP-13, but rather MMP-2, whose substrate specificity includes collagen IV and denatured type I collagen (gelatin) $(4,33)$. Moreover, upregulation of MMP-2 has been observed in cultured stellate cells in response to type I collagen, but the underlying mechanism has not been identified $(22,34,35)$.

We therefore examined whether DDR2 might mediate type I collagen s ability to stimulate MMP-2 expression by stellate cells. Western blot of culture supernatants demonstrated that MMP-2 expression was increased in HSC-T6 cells expressing the constitutively active Fc-DDR2 chimera and was significantly reduced
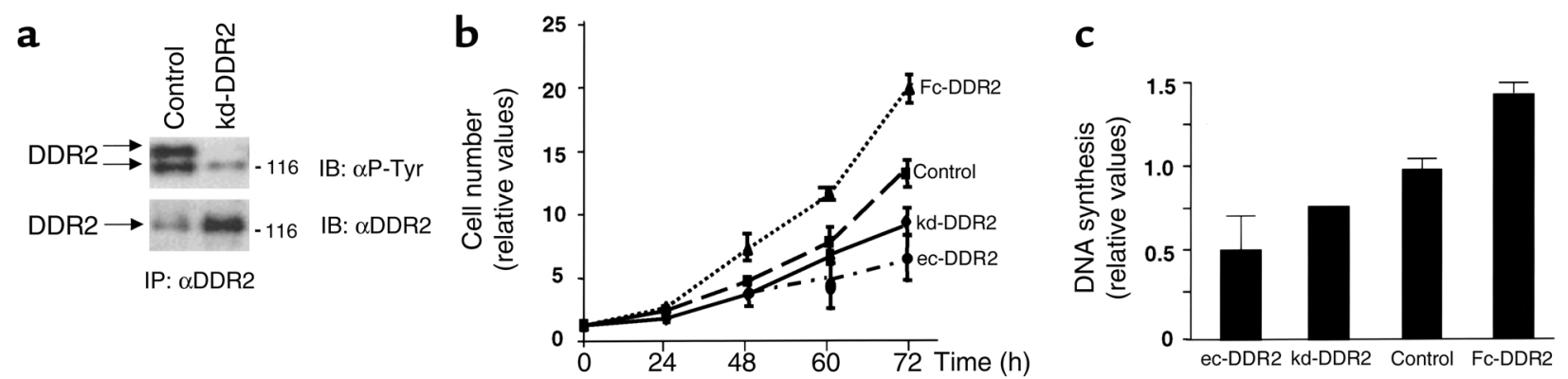

Figure 6

DDR2 promotes HSC proliferation. HSC-T6 cells were infected with a retrovirus containing a bi-cistronic cDNA expressing either a truncated form of the receptor lacking the kinase domain (ec-DDR2), a kinase-dead mutant (kd-DDR2), GFP (control), or a constitutively active DDR2 receptor (Fc-DDR2). Infected cells were recovered by cell sorting for GFP fluorescence using FACS. (a) Retroviral infection with kd-DDR2 inhibits the phosphorylation of the endogenous DDR2 receptor in response to a 30 -minute exposure to $10 \mu \mathrm{g} / \mathrm{ml}$ collagen type I. Proliferation rates were analyzed by measuring $(\mathbf{b})\left[{ }^{3} \mathrm{H}\right]$-thymidine incorporation or $(\mathbf{c})$ cell number and expressed as relative values versus control cells. 
a

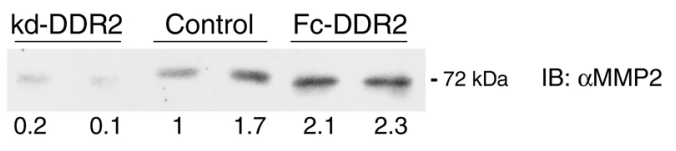

b

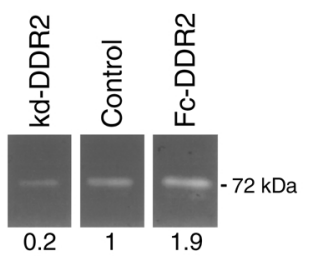

\section{Figure 7}

DDR2 promotes MMP-2 expression and activity in stellate cells. Serum-free supernatants from HSC-T6 cells expressing a kinase-dead mutant of the receptor (kd-DDR2), GFP alone (control), or the constitutively active Fc-DDR2 were analyzed in triplicate experiments for (a) MMP-2 protein using Western blots and (b) MMP-2 activity by gelatin zymography. Relative expression was quantitated by the NIH Image software and shown beneath each lane.

in cells expressing the mutant kd-DDR2, as compared with control cells expressing only GFP (Figure 7a). Zymography assay revealed a band of gelatin degradation at $72 \mathrm{kDa}$, representing latent MMP-2, and a faint band of $68 \mathrm{kDa}$, representing active MMP-2 (Figure $7 \mathrm{~b})$. Cells expressing ec-DDR2 also had reduced MMP-2 expression and activity (data not shown). Gelatinase activity was confined to the medium and none was found in cell lysates (not shown).

Gelatinase activity was also demonstrated using a colorimetric-based system and expressed as $1 / \mathrm{OD}_{450}$ (see Methods). Gelatinase activity could be ascribed to MMP-2 because conditioned media from HSC-T6 cells did not contain MMP-9 activity (as assessed by gelatin zymography). Gelatinase activity in the conditioned media from control and Fc-DDR2-expressing cells was $5-10 \%$ of that present in the APMA-activated conditioned media $\left(1 / \mathrm{OD}_{450}=10\right.$ for APMA-activated conditioned media from control cells). HSC-T6 cells expressing Fc-DDR2 expressed 80\% more gelatinase activity than control cells $\left(1 / \mathrm{OD}_{450}=1\right.$ non-APMAactivated conditioned media from control cells and $1 / \mathrm{OD}_{450}=1.8$ for non-APMA-activated conditioned media from Fc-DDR2-expressing cells). Gelatinase activity in the conditioned media from ecDDR2-expressing cells was below the level of detection.

Recent studies have suggested that MMP-2 is mitogenic for hepatic stellate cells (HSCs) (36). Therefore, we examined whether the enhanced proliferation of stellate cells expressing the constitutively active Fc-DDR2 was directly related to their increased activity of MMP-2. To do so, MMP-2 activity was inhibited by adding incremental concentrations of recombinant TIMP-2, the natural inhibitor of MMP-2. Addition of TIMP-2 inhibited cell proliferation in a dose-dependent manner. Moreover, cells expressing the Fc-DDR2 required higher concentrations of TIMP-2 to achieve levels of inhibition comparable to those of control cells
(Figure 8a). Similar results were obtained using either of two recombinant TIMP-2 sources. Addition of the MMP inhibitor GM6001 $(10 \mu \mathrm{M})$ resulted in a lesspronounced inhibition of cell proliferation in the Fc-DDR2 than in the control cells (Figure 8a). Gelatin zymography and a gelatinase activity assay of media conditioned for 12 hours by control cells demonstrated that $10 \mu \mathrm{M}$ GM6001 did not affect the levels of MMP-2 (result not shown), while it inhibited $75 \%$ of its gelatinolytic activity (data not shown).

TIMP-2 and MT1-MMP, along with MMP-2, comprise a trimolecular complex that collectively regulates MMP-2 activity (6). TIMP-2 and MT1-MMP levels were similar between the Fc-DDR2-expressing cells and the control cells (Figure $8 \mathrm{~b}$ ), suggesting that the enhanced proliferation of stellate cells expressing the constitutively active Fc-DDR2 was directly related to their increased activity of MMP-2.

$D D R 2$ promotes migration through basement membrane by stellate cells. A critical response of activated stellate cells during liver injury is their migration to regions of inflammation and necrosis. To migrate, stellate cells must degrade the subendothelial matrix, which is rich in collagen IV, the principal substrate for MMP-2 (37), although degradative activity against type I collagen has also been reported (38). To study the importance of DDR2 in cell migration, stellate cells expressing active or inactive DDR2 were assayed for their capacity to migrate through a Matrigel-coated insert in response to a chemotactic stimulus. Expression of a truncated form

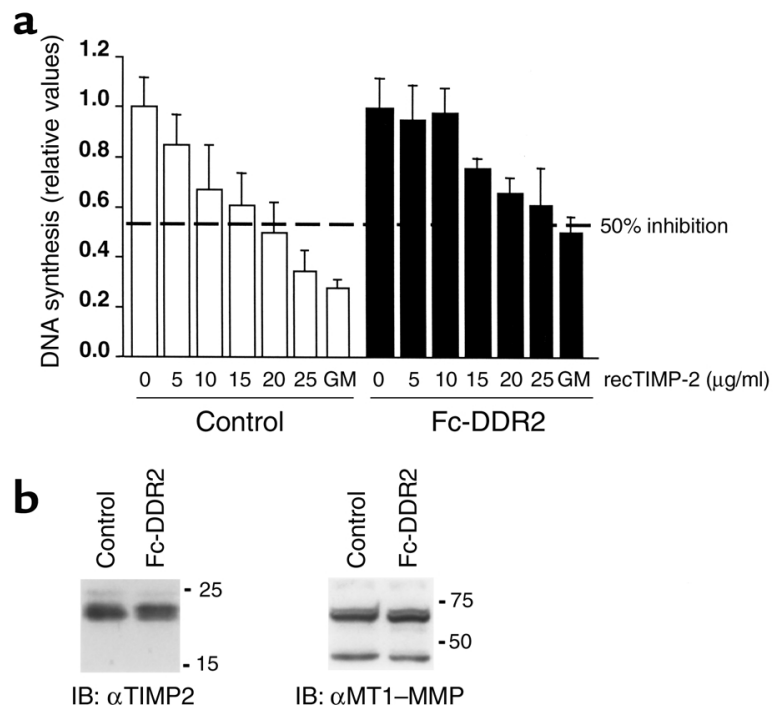

Figure 8

Increased TIMP-2 or the MMP inhibitor GM6001 are required to inhibit proliferation of stellate cells overexpressing DDR2. (a) HSC-T6 cells expressing GFP alone (control) or the constitutively active Fc-DDR2 were cultured for 48 hours in DMEM with $1 \%$ FCS in the presence of recombinant TIMP-2 or GM6001, $10 \mu \mathrm{M}$ (GM). Incorporation of $\left[{ }^{3} \mathrm{H}\right]$-thymidine during the last 6 hours of the experiment was measured. (b) TIMP-2 and MT1-MMP expression were analyzed by Western blots in the serum-free supernatants, or in the lysates from HSC-T6 cells expressing GFP alone (control), or Fc-DDR2. 
a

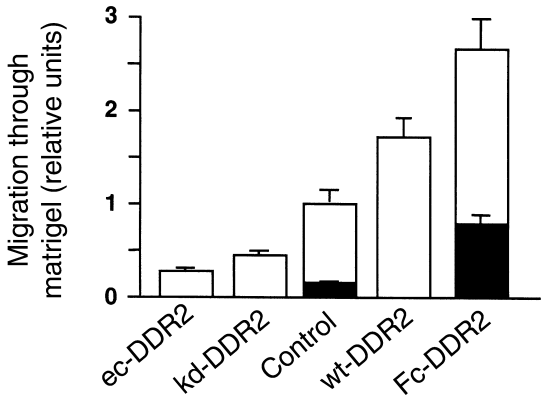

b

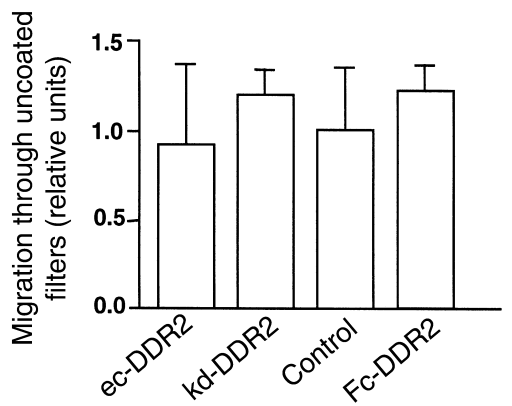

\section{Figure 9}

DDR2 promotes invasion into basement membrane matrix by stellate cells and is inhibited by GM6001. (a) A representative experiment of HSC-T6 cells infected with the ec-DDR2, kd-DDR2, GFP alone (control), wt-DDR2, or the constitutively active Fc-DDR2 were suspended in DMEM plus 1\% FCS and placed on inserts $(8-\mu \mathrm{m}$ pore size) coated with $100 \mu \mathrm{g} / \mathrm{cm}^{2}$ of Matrigel. Conditioned media from Kupffer cell cultures supplemented with 1\% FCS was used as chemoattractant. After 12 hours, migrating cells adhering to the underside of the membrane were stained with $0.2 \%$ crystal violet and counted in ten random high-power fields per insert. Filled columns represent migration of HSC-T6 cells pretreated with $20 \mu \mathrm{M}$ GM6001 for 1 hour, then allowed to migrate in the presence of $10 \mu \mathrm{M}$ GM6001. The number of control cells that had migrated to the lower chamber after 12 hours was $55 \pm 6$ per high-power field. (b) A representative experiment of HSC-T6 cells migrating for 4 hours through uncoated inserts. The number of control HSC-T6 cells that had migrated to the lower chamber was $293 \pm 80$ per high-power field.

lacking the kinase domain (ec-DDR2) or a kd-DDR2 significantly reduced invasion through a Matrigel-coated insert compared with control cells expressing only GFP (see Methods). In contrast, overexpression of wtDDR2 or expression of the constitutively active Fc-DDR2 increased cell migration through Matrigel when compared with control cells (Figure 9a). Migration through Matrigel correlated with the levels of active MMP-2 secreted into the media as assessed by zymography (Figure $7 \mathrm{~b}$ ) and gelatinase activity assay (see above). Gelatinase activity in the conditioned media from control cells cultured under the conditions used for cell migration (i.e., 12-hour culture onto Matrigel, $100 \mu \mathrm{g} / \mathrm{cm}^{2}$ ) was the same as when cultured on plastic. Addition of the MMP inhibitor GM6001 $(10 \mu \mathrm{M})$ inhibited by $64 \%$ the migration of Fc-DDR2-expressing cells and by $80 \%$ the migration of control cells (Figure 9a).

In contrast to these results on Matrigel, which reflect migration, there was no consistent effect of
DDR2 when cells were placed on uncoated filters, indicating that DDR2 affects invasion but not generalized motility (Figure 9b).

\section{Discussion}

Our data demonstrate that expression of DDR2 accompanies activation of stellate cells in vivo and in culture and is regulated by extracellular matrix composition. Moreover, DDR2 is restricted to stellate cells among resident liver cell types, consistent with the observation that DDR2 expression is confined to mesenchymal cell lineages (14). Furthermore, the findings provide evidence of a positive feedback loop in which induction of DDR2 leads to enhanced cell proliferation and MMP-2 activity, with accelerated degradation of a collagen type IV-containing matrix. This could allow for replacement of the hepatic subendothelial matrix by fibrillar collagen, which in turn is the ligand for DDR2. The data further explain how interstitial collagen in the pericellular milieu directly provokes stellate cell activation through specific receptor signaling by DDR2.

In the context of the two-stage paradigm of stellate cell activation (1), DDR2 perpetuates rather than initiates the fibrotic response for the following reasons: first, peak induction of DDR2 mRNA in stellate cells occurs relatively late compared with other RTKs. For example, DDR $2 \mathrm{mRNA}$ is maximal only 48 hours after $\mathrm{CCl}_{4}$ administration in vivo or after 8 days in culture, whereas $\beta$-PDGF receptor $\mathrm{mRNA}$ is detectable within 3 hours after $\mathrm{CCl}_{4}$ in vivo and is maximal at 24 hours or within 3 days after plating in primary culture (8). Second, DDR2 phosphorylation occurs more slowly than other RTKs or integrins (15). In contrast to integrins that lead to FAK phosphorylation within minutes in response to collagen (39), DDR2 phosphorylation in response to collagen occurs only after 2 hours. Third, no significant downregulation of DDR2 phosphorylation is observed for several days (result not shown). This "slow-on, slow-off" response to ligand suggests that once activated, DDR2 signaling could provide a relatively sustained stimulus to matrix degradation and stellate cell proliferation.

Based on the temporal sequence of events during stellate cell activation, DDR2 phosphorylation occurs at a point when cells are already synthesizing high levels of collagen I and thus can provide ample autocrine ligand. This conclusion is supported by our finding that specific inhibition of endogenous collagen synthesis downregulated DDR2 phosphorylation. Thus, the relative concentration of ligand may be an important determinant of DDR2 activity. This is consistent with the observation that although DDR2 is detectable in normal liver, the phosphorylated receptor is only detectable in injured liver, where ligand becomes available.

Our findings also illustrate that the extracellular matrix composition has a dominant regulatory effect on stellate cell activation, not only determining DDR2 expression, but also other features of activation such as morphology, cell growth, and smooth 
muscle actin expression. Previously, we demonstrated that freshly isolated stellate cells cultured in a basement membrane-like matrix remain quiescent, while those cultured on collagen I lattices are activated (3). In the current study, however, even a transformed, highly activated rat stellate cell line can become quiescent when transferred to Matrigel. Downregulation of DDR2 by Matrigel is concentration and time dependent, with a threshold amount of matrix required to induce quiescence (data not shown). The quiescent effect of Matrigel on activated stellate cells is reversible and not due to apoptosis, because cells replated on plastic proliferate at the same rate as those maintained throughout on plastic. The mechanism by which basement membrane matrix preserves quiescence is not yet clear, but the implication is that even activated cells in injured liver may harbor the capacity to revert to a quiescent phenotype if the normal extracellular matrix can be restored.

We explored the role of DDR2 in regulating metalloproteinase activity in stellate cells because DDR2 has been associated with increased MMP-1 activity in the human fibrosarcoma cell line HT 1080 (15); primary cultures of stellate express only MMP-2 and not MMP-1 $(4,33)$. HSC-T6 cells express neither MMP-13 nor murine MMP-1; however, it remains possible that other unidentified MMPs could also be regulated by DDR2 signaling in stellate cells. DDR2 appears to control MMP-2 activity at several levels. In skin fibroblasts, MMP-2 promoter activity requires DDR2 signaling (unpublished data). In stellate cells, MMP-2 expression and activity are also tied to DDR2 signaling, since MMP-2 expression and activity were reduced in cells expressing a nonsignaling DDR2. However, the addition of soluble collagen I to kd-DDR2-expressing stellate cells still promoted a minor increase in MMP-2 expression (result not shown). This suggests that other pathways independent of DDR2 may also influence MMP-2 expression. In accordance with this possibility, integrin signaling via collagens increases MMP-2 activity in human stellate cells (21).

Net MMP-2 collagenase activity is also tightly regulated by its relative stoichiometry with MT1-MMP and TIMP-2 (6). In our stellate cell system, however, MT1MMP and TIMP-2 levels remained unchanged regardless of DDR2 expression levels, suggesting that MMP-2 activity most closely correlates with relative concentrations of the enzyme. In turn, the growth-promoting effect of DDR2 on stellate cells is closely tied to MMP-2 activity, since addition of TIMP-2 abrogated proliferation. Although we cannot exclude a direct effect of TIMP-2 on growth suppression independent of MMP-2 (40), or one mediated by another MMP, the inhibition of growth was also observed in the presence of other MMP inhibitors, GM6001 and 1,10 phenanthroline (result not shown), similar to the report of Benyon (36). These findings suggest that MMP-2 regulates growth in stellate cells directly. Moreover, increased concentrations of the inhibitors TIMP-2 or GM6001 were required to inhibit prolifera- tion in stellate cells overexpressing DDR2 to the same extent as that of control cells. The effect of GM6001 is due to direct inhibition of MMP-2 activity and not expression. Also, recent reports indicated that TIMP- 2 does not affect MMP-2 expression in stellate cells (22) or human vascular endothelial cells (41). Although the exact mechanism of growth regulation by MMP-2 is uncertain, it might cleave and/or activate growth-promoting factor(s) in the extracellular milieu, or even bind directly to a cell surface receptor, as has been demonstrated for other proteases, including protein S (42). However, not all cell proliferation can be ascribed to MMP-2, since some proliferation persisted even in the presence of high levels of exogenous TIMP-2 or GM6001. MMP-2-independent, direct proliferative signals mediated through the DDR2 receptor are possible and may become apparent as the intracellular signaling pathways are subsequently revealed. Evidence to date has identified Src kinase and the adaptor molecular Shc as a key signaling intermediates immediately downstream of the DDR2 receptor (K. Ikeda, manuscript submitted for publication). Future studies will explore their potential interactions with signaling pathways that promote growth and/or cell cycle progression. Finally, other as yet unidentified MMPs also regulated by DDR2 could serve as growth factors for HSCs.

An emerging theme in signaling is the importance of cooperating lateral networks involving heterologous families of cell surface receptors (43). Thus, it would be tempting to speculate that DDR2 might associate with integrins in binding collagen ligand. However, to date neither we nor others have documented such a direct association for either DDR1 (44) or DDR2 (K. Ikeda, manuscript submitted for publication). While DDR1 does not colocalize with integrin receptors, it has been proposed that DDR2 and integrin signaling pathways may converge further downstream (44). This convergence might involve Shc, since this molecule has also been implicated in integrin signaling as well as that of DDR1 (44) and DDR2 (K. Ikeda, manuscript submitted for publication).

A key event in progressive liver injury is the migration of activated stellate cells to regions of injury, typically along forming septa (45). Migration in vivo requires a chemoattractant stimulus, degradative (i.e., proteolytic) activity to erode surrounding pericellular matrix, as well as associated changes in cytoskeleton and membrane polarity to support directional movement (37). In these studies chemoattraction was provided by Kupffer cell-conditioned medium, a highly physiologic stimulus given their rapid accumulation in vivo prior to stellate cell migration (45). The DDR2-mediated migration through basement membrane by activated stellate cells also contributes to their accumulation in sites where injury is greatest. Like its effects on proliferation, DDR2's stimulation of basement membrane invasion by stellate cells likely requires active MMP-2, although MMP-2-independent effects on cytoskeleton and membrane activity required for invasiveness remain to be elucidated. DDR2 may serve a simi- 
lar role in tumor invasion, where it has been localized to stromal cells surrounding tumors of epithelial origin (14). In this regard, regulation of murine MMP-1 (46) and MMP-13 mRNAs by endogenous DDR2 signaling in tumor-associated myofibroblasts and its effect on their proliferation and migration are under study.

In summary, our findings support a model in which induction and phosphorylation of DDR2 during activation of stellate cells stimulates key events that perpetuate the activated phenotype, including cell growth, matrix degradation, and basement membrane invasion. Our data also indicate that all these events can be downregulated by restoring a normal extracellular milieu rich in basement membrane collagen. Collectively, the evidence points to a central role of DDR2 in liver fibrosis and the prospect of altering the fibrotic response by interfering with DDR2 expression and/or activity.

\section{Acknowledgments}

E. Olaso is a recipient of a research grant from the Basque government. The work was supported by grants from the NIH (DK-56621) and the Mount Sinai School of Medicine Dean's research incentive fund. The authors gratefully acknowledge the technical assistance of Qiang Liu and the members of the INBIOMED Foundation.

1. Friedman, S.L. 2000. Molecular regulation of hepatic fibrosis; an integrated cellular response to tissue injury. J. Biol. Chem. 275:2247-2250.

2. Olaso, E., and Friedman, S.L. 1998. Molecular regulation of hepatic fibrogenesis. J. Hepatol. 29:836-847.

3. Friedman, S.L., Roll, F.J., Boyles, J., Arenson, D.M., and Bissell, D.M. 1989. Maintenance of differentiated phenotype of cultured rat hepatic lipocytes by basement membrane matrix. J. Biol. Chem. 264:10756-10762.

4. Arthur, M.J., et al. 1992. Secretion of $72 \mathrm{kDa}$ type IV collagenase/gelatinase by cultured human lipocytes. Analysis of gene expression, protein synthesis and proteinase activity. Biochem. J. 287:701-707.

5. Butler, G.S., et al. 1998. The TIMP2 membrane type 1 metalloproteinase receptor regulates the concentration and efficient activation of progelatinase A. J. Biol. Chem. 273:871-880.

6. Werb, Z. 1997. ECM and cell surface proteolysis: regulating cellular ecology. Cell. 91:439-442.

7. Pinzani, M., Marra, F., and Carloni, V. 1998. Signal transduction in hepatic stellate cells. Liver. 18:2-13.

8. Wong, L., Yamasaki, G., Johnson, R.J., and Friedman, S.L. 1994. Induction of beta-platelet-derived growth factor receptor in rat hepatic lipocytes during cellular activation in vivo and in culture. J. Clin. Invest. 94:1563-1569.

9. Bachem, M.G., Riess, U., and Gressner, A. 1989. Liver fat storing cell proliferations stimulated by epidermal growth factor/transforming growth factor alpha and inhibited by transforming growth factor beta. Biochem. Biophys. Res. Commun. 162:708-714.

10. Rosenbaum, J., et al. 1995. Fibroblast growth factor 2 and transforming growth factor beta 1 interactions in human liver myofibroblasts. Gastroenterology. 109:1986-1996.

11. Ankoma-Sey, V., et al. 1998. Coordinated induction of VEGF receptors in mesenchymal cell types during rat hepatic wound healing. Oncogene. 17:115-121.

12. Lai, C., and Lemke, G. 1994. Structure and expression of the Tyro 10 receptor tyrosine kinase. Oncogene. 9:877-883.

13. Karn, T., et al. 1993. Structure, expression and chromosomal mapping of TKT from man and mouse: a new subclass of receptor tyrosine kinase with a factor VIII-like domain. Oncogene. 8:3433-3440.

14. Alves, F., et al. 1995. Distinct structural characteristics of discoidin I subfamily receptor tyrosine kinases and complementary expression in human cancer. Oncogene. 10:609-618.

15. Vogel, W., Gish, G.D., Alves, F., and Pawson, T. 1997. The discoidin domain receptor tyrosine kinases are activated by collagen. Mol. Cell. 1:13-23.

16. Shrivastava, A., et al. 1997. An orphan receptor tyrosine kinase family whose members serve as nonintegrin collagen receptors. Mol. Cell. 1:25-34.

17. Barker, K.T., et al. 1995. Expression patterns of the novel receptor-like tyrosine kinase, DDR, in human breast tumours. Oncogene. 10:569-575.

18. Hynes, R.O. 1992. Integrins: versatility, modulation and signalling in cell adhesion. Cell. 69:11-25.

19. Carloni, V., Romanelli, R.G., Pinzani, M., Laffi, G., and Gentilini, P. 1996 Expression and function of integrin receptors for collagen and laminin in cultured human hepatic stellate cells. Gastroenterology. 110:1127-1136.

20. Racine-Samson, L., Rockey, D.C., and Bissell, D.M. 1997. The role of alpha1beta 1 integrin in wound contraction. A quantitative analysis of liver myofibroblasts in vivo and in primary culture. J. Biol. Chem. 272:30911-30917

21. Carloni, V., Romanelli, R.G., Pinzani, M., Laffi, G., and Gentilini, P. 1997. Focal adhesion kinase and phospholipase $C$ gamma involvement in adhesion and migration of human hepatic stellate cells. Gastroenterology. 112:522-531.

22. Theret, N., Lehti, K., Musso, O., and Clement, B. 1999. MMP2 activation by collagen I and concanavalin A in cultured human hepatic stellate cells. Hepatology. 30:462-468.

23. Friedman, S. 1993. Isolation and culture of hepatic nonparenchymal cells. In In vitro biological systems. Volume 1. C. Tyson and J. Frazier, editors. Academic Press. San Diego, California, USA. 292-310.

24. Vogel, S., et al. 2000. An immortalized rat liver stellate cell line (HSC-T6): a new cell model for the study of retinoid metabolism in vitro. J. Lipid. Res. 41:882-893.

25. Proctor, E., and Chatamra, K. 1982. High yield micronodular cirrhosis in the rat. Gastroenterology. 83:1183-1190.

26. Kountouras, J., Billing, B.H., and Scheuer, P.J. 1984. Prolonged bile duct obstruction: a new experimental model for cirrhosis in the rat. Br.J. Exp. Pathol. 65:305-311.

27. Greenwel, P., Rubin, J., Schwartz, M., Hertzberg, E.L., and Rojkind, M.. 1993. Liver fat-storing cell clones obtained from a CCl4-cirrhotic rat are heterogeneous with regard to proliferation, expression of extracellular matrix components, interleukin-6, and connexin 43. Lab. Invest. 69:210-216

28. Olaso, E., et al. 1997. Tumor-dependent activation of rodent hepatic stellate cells during experimental melanoma metastasis. Hepatology. 26:634-642.

29. Albini, A., et al. 1986. A rapid in vitro assay for quantitating the invasive potential of tumor cells. Cancer Res. 47:3239-3245.

30. Maher, J.J., and McGuire, R.F. 1990. Extracellular matrix gene expression increases preferentially in rat lipocytes and sinusoidal endothelial cells during hepatic fibrosis in vivo. J. Clin. Invest. 86:1641-1648.

31. Friedman, S.L., Roll, F.J., Boyles, J., and Bissell, D.M. 1985. Hepatic lipocytes: the principal collagen-producing cells of normal rat liver. Proc. Natl. Acad. Sci. USA. 82:8681-8685.

32. Rocnik, E.F., Chan, B.M.C., and Pickering, J.G. 1998. Evidence for a role of collagen synthesis in arterial smooth muscle cell migration. J. Clin. Invest. 101:1889-1898.

33. Arthur, M.J., Friedman, S.L., Roll, F.J., and Bissell, D.M. 1989. Lipocytes from normal rat liver release a neutral metalloproteinase that degrades basement membrane (type IV) collagen. J. Clin. Invest. 84:1076-1085.

34. Theret, N., Musso, O., L'Helgoualc'h, A., and Clement, B. 1997. Activation of matrix metalloproteinase-2 from hepatic stellate cells requires interactions with hepatocytes. Am. J. Pathol. 150:51-58.

35. Takahara, T., et al. 1995. Increased expression of matrix metalloproteinase-II in experimental liver fibrosis in rats. Hepatology. 21:787-795

36. Benyon, R.C., et al. 1999. Progelatinase A is produced and activated by rat hepatic stellate cells and promotes their proliferation. Hepatology. 30:977-986.

37. Ikeda, K., et al. 1999. In vitro migratory potential of rat quiescent hepatic stellate cells and its augmentation by cell activation. Hepatology. 29:1760-1767.

38. Aimes, R.T., and Quigley, J.P. 1995. Matrix metalloproteinase-2 is an interstitial collagenase. Inhibitor- free enzyme catalyzes the cleavage of collagen fibrils and soluble native type I collagen generating the specific $3 / 4$ and 1/4-length fragments. J. Biol. Chem. 270:5872-5876.

39. Sieg, D.J., et al. 2000. FAK integrates growth-factor and integrin signals to promote cell migration. Nat. Cell Biol. 2:249-255.

40. Hoegy, S.E., Oh, H.-R., Corcoran, M.L., and Stetler-Stevenson, W.G. 2001 Tissue inhibitor of metalloproteinases-2 (TIMP-2) suppresses TKR growth factor signaling independent of metalloproteinase inhibition. $J$. Biol. Chem. 276:3203-3321.

41. Lewalle, J.M., et al. 1995. Plasma membrane-dependent activation of gelatinase A in human vascular endothelial cells. J. Cell Physiol. 165:475-483.

42. Stitt, T.N., et al. 1995. The anticoagulation factor protein $\mathrm{S}$ and its relative, Gas6, are ligands for the Tyro 3/Axl family of receptor tyrosine kinases. Cell. 80:661-670.

43. Pinkas-Kramarski, R., et al. 1996. Diversification of Neu differentiation factor and epidermal growth factor signaling by combinatorial receptor interactions. EMBO J. 15:2452-2467.

44. Vogel, W., et al. 2000. Discoidin domain receptor 1 is activated independently of beta1 integrin. J. Biol. Chem. 275:5779-5784.

45. Johnson, S.J., Hines, J.E., and Burt, A.D. 1992. Macrophage and perisinusoidal cell kinetics in acute liver injury. J. Pathol. 166:351-358.

46. Balbin, M., et al. 2001. Identification and enzymatic characterization of two diverging murine counterparts of human interstitial collagenase (MMP-1) expressed at the site of embryo implantation. J. Bio. Chem. 276:10253-10262. 